\title{
Three-Dimensional Reconstruction of Trypanosoma brucei Editosomes Using Single-Particle Electron Microscopy
}

\author{
H. Ulrich Göringer, Holger Stark, Cordula Böhm, Bjoern Sander, \\ and Monika M. Golas
}

\begin{abstract}
RNA editing within the mitochondria of kinetoplastid protozoa is performed by a multicomponent macromolecular machine known as the editosome. Editosomes are high molecular mass protein assemblies that consist of about 15-25 individual polypeptides. They bind pre-edited transcripts and convert them into translation-competent mRNAs through a biochemical reaction cycle of enzyme-catalyzed steps. At steady-state conditions, several distinct complexes can be purified from mitochondrial detergent lysates. They likely represent RNA editing complexes at different assembly stages or at different functional stages of the processing reaction. Due to their low cellular abundance, single-particle electron microscopy (EM) represents the method of choice for their structural characterization. This chapter describes a set of techniques suitable for the purification and structural characterization of RNA editing complexes by singleparticle EM. The RNA editing complexes are isolated from the endogenous pool of mitochondrial complexes by tandem-affinity purification (TAP). Since the TAP procedure results in the isolation of a mixture of different RNA editing complexes, the isolates are further subjected to an isokinetic ultracentrifugation step to separate the complexes based on their sedimentation behavior. The use of the "GraFix" protocol is presented that combines mild chemical cross-linking with ultracentrifugation. Different sample preparation protocols including negative staining, cryo-negative staining, and unstained cryotechniques as well as the single-particle image processing of electron microscopical images are described.
\end{abstract}

Key words: RNA editing, Editosome, Trypanosoma brucei, Tandem-affinity purification (TAP), GraFix, Surface plasmon resonance (SPR), Density gradient centrifugation, Electron microscopy (EM), Cryo-EM, Single-particle image processing

\section{Introduction}

Many cellular proteins act in complexes with other proteins or nucleic acids as part of high molecular mass macromolecular machines (1). These complexes typically assemble in multistep reaction pathways, and thus the composition and function of 
complexes largely depends on the assembly step. One such example is the RNA editing machinery in kinetoplastid protozoa such as African trypanosomes and Leishmania. The RNA editing complexes generate messenger ribonucleic acid (mRNA) molecules from immature pre-messenger RNA (pre-mRNA) by the insertion and/or deletion of exclusively uridylate residues $(2,3)$. In trypanosomes, more than 20 proteins are involved in the process and the catalytic complexes have been termed as editosomes. Different RNA editing complexes sedimenting between about 5 and 40 Svedberg units (S) have been identified.

For the three-dimensional (3D) structural analysis of such multicomponent complexes, single-particle transmission electron microscopy (EM) represents the method of choice $(4,5)$. In contrast to X-ray crystallography and nuclear magnetic resonance (NMR), single-particle EM can successfully deal with low sample concentrations (in case of the editosomes typically below $10 \mu \mathrm{g} / \mathrm{ml}$ ) even in the case of transient and fragile assemblies (6). The basic principle of single-particle EM is the computational merging of many thousands of individual particles imaged in the electron microscope to reconstruct their $3 \mathrm{D}$ structure $(7,8)$. Due to their pronounced radiation sensitivity, biological samples have to be imaged at low-dose conditions. As a consequence, the raw EM images are noisy and signals need to be enhanced by computational averaging methods. In addition, the structural analysis of macromolecular assemblies is often hampered by the disintegration and/or aggregation of the complexes during purification and sample preparation, thereby limiting the applicability of singleparticle EM on a number of macromolecular machines. However, a novel approach offers a promising protocol to tackle these limitations (9). Recent developments in single-particle EM allow the analysis of structural transitions and dynamics processes during the assembly and catalytic cycle of macromolecular machines on the 3D level.

\section{Materials}

\subsection{TAP Purification of RNA Editing Complexes}

1. SDM-79 medium (all media components from Invitrogen, Karlsruhe, Germany) supplemented with $10 \%(\mathrm{v} / \mathrm{v})$ fetal calf serum $(10)$.

2. pLEW100/TAP, a derivative of pLEW100 (11).

3. Primers used to amplify the coding region of TbMP42 (GenBankAF382335):TbMP42-5'-primer:CCGCTCGAGAT GAAGCGTGTTACTTCACATATTTCG;TbMP42-3'-primer: TGCTCTAGACACCCTCAACACTGACCCAAGCC. 
4. Editing buffer: $20 \mathrm{mM}$ HEPES-KOH, pH 7.5, $30 \mathrm{mM} \mathrm{KCl}$, $10 \mathrm{mM} \mathrm{Mg}(\mathrm{OAc})_{2}, 0.5 \mathrm{mM}$ DTT.

5. Lysis buffer: $20 \mathrm{mM}$ HEPES-KOH pH 7.5, $30 \mathrm{mM} \mathrm{KCl}$, $10 \mathrm{mM} \mathrm{Mg}(\mathrm{OAc})_{2}, 0.5 \mathrm{mM}$ DTT, 0.6\% (v/v) Nonidet-P40.

6. IPP150: 10 mM Tris- $\mathrm{HCl}, \mathrm{pH} 8,150 \mathrm{mM} \mathrm{NaCl}, 1 \%(\mathrm{v} / \mathrm{v})$ Nonidet-P40.

7. IgG Sepharose (Amersham Biosciences, Freiburg, Germany) equilibrated in editing buffer.

8. TEV cleavage buffer: $10 \mathrm{mM}$ Tris- $\mathrm{HCl}, \mathrm{pH} 8,150 \mathrm{mM}$ $\mathrm{NaCl}, 1 \%$ (v/v) Nonidet-P40, $0.5 \mathrm{mM} \mathrm{Na}{ }_{2}$ EDTA, $1 \mathrm{mM}$ DTT.

9. TEV protease (BioPioneer Inc., San Diego, CA, USA).

10. Calmodulin binding buffer: $10 \mathrm{mM}$ Tris- $\mathrm{HCl}, \mathrm{pH} 8,150 \mathrm{mM}$ $\mathrm{NaCl}, 1 \%$ (v/v) Nonidet-P40, 10 mM 2-mercaptoethanol, $1 \mathrm{mM} \mathrm{Mg}(\mathrm{OAc})_{2}, 1 \mathrm{mM}$ imidazol, $2 \mathrm{mM} \mathrm{CaCl}_{2}$.

11. Calmodulin affinity resin (Stratagene, La Jolla, USA) equilibrated in editing buffer.

12. Calmodulin elution buffer: $10 \mathrm{mM}$ Tris- $\mathrm{HCl}, \mathrm{pH} 8,150 \mathrm{mM}$ $\mathrm{NaCl}, 1 \%$ (v/v) Nonidet-P40, $10 \mathrm{mM} 2$-mercaptoethanol, $1 \mathrm{mM} \mathrm{Mg}(\mathrm{OAc})_{2}, 1 \mathrm{mM}$ imidazol, 5 mM EGTA.

13. RNasin(C.

2.2. Biochemical Analysis
1. Calf intestine phosphatase.

2. Guanylyl transferase (Ambion Inc., Austin, TX, USA).

3. Polynucleotide kinase.

4. $\gamma-\left[{ }^{32} \mathrm{P}\right]$-ATP.

5. $\alpha-\left[{ }^{32} \mathrm{P}\right]-\mathrm{GTP}$.

6. $\mathrm{Na}\left[{ }^{125} \mathrm{I}\right]$ (Hartmann Analytic). See Note 1.

7. Chloramine-T (Acros Organics, Geel, Belgium) dissolved in $50 \mathrm{mM}$ sodium phosphate, $\mathrm{pH}$ 7.5.

8. $\mathrm{Na}_{2} \mathrm{~S}_{2} \mathrm{O}_{5}$ (Acros Organics, Geel, Belgium) dissolved in $50 \mathrm{mM}$ sodium phosphate, $\mathrm{pH}$ 7.5.

9. Beckman TLS55 rotor.

10. Low percentage buffer: 10\% (v/v) glycerol, $20 \mathrm{mM}$ HEPES$\mathrm{KOH} \mathrm{pH} 7.5,30 \mathrm{mM} \mathrm{KCl}, 10 \mathrm{mM} \mathrm{Mg}(\mathrm{OAc})_{2}, 0.5 \mathrm{mM}$ DTT.

11. High percentage buffer: 40\% (v/v) glycerol, $20 \mathrm{mM}$ HEPES$\mathrm{KOH}, \mathrm{pH} 7.5,30 \mathrm{mM} \mathrm{KCl}, 10 \mathrm{mM} \mathrm{Mg}(\mathrm{OAc})_{2}, 0.5 \mathrm{mM}$ DTT, $0.1 \%(\mathrm{v} / \mathrm{v})$ glutaraldehyde.

12. Cushion buffer: $20 \mathrm{mM}$ HEPES-KOH pH 7.5, $30 \mathrm{mM} \mathrm{KCl,}$ $10 \mathrm{mM} \mathrm{Mg}(\mathrm{OAc})_{2}, 0.5 \mathrm{mM}$ DTT, 10\% (v/v) glycerol. 
2.3. Surface Plasmon Resonance

\subsection{GraFix Fractionation}

\subsection{Negative Staining and Cryo-negative Staining}

1. Oxidation buffer: $50 \mathrm{mM} \mathrm{NaOAc}, \mathrm{pH} 4.8,10 \mathrm{mM} \mathrm{MgCl}$, $100 \mathrm{mM} \mathrm{NaCl}$.

2. Coupling buffer: $100 \mathrm{mM}$ sodium phosphate, $\mathrm{pH} 7.2$, $150 \mathrm{mM} \mathrm{NaCl}, 50 \mathrm{mM} \mathrm{NaBH} \mathrm{m}_{3} \mathrm{CN}$.

3. IAsys surface plasmon resonance (SPR) instrument (NeoSensors, Sedgefield, UK).

4. Aminosilane-coated SPR reaction cuvette (NeoSensors, Sedgefield, UK).

1. Gradient preparation device, e.g., GradientMaster (Biocomp, Fredericton, NB, Canada).

2. Ultracentrifuge, rotors and centrifugation tubes: Sorvall or Beckman ultracentrifuge with suitable rotor with a capacity of about $13.2 \mathrm{ml}$ per centrifugation tube (e.g., Sorvall TH-64l).

3. Gradient fractionation device: custom-made needle/tube system connected to a peristaltic pump and a UV spectrophotometer (GE Healthcare, Buckinghamshire, UK) or tube piercing device (Brandel Isco tube piercer, Isco, Lincoln, USA).

4. EM-grade $25 \%(\mathrm{v} / \mathrm{v})$ glutaraldehyde solution (Electron Microscopy Sciences, Hatfield, PA, USA). See Note 2.

1. Custom-made black plastic (polyoxymethylene) or Teflon block with holes of about 25-30 and 100-200 $\mu$ l volume.

2. Home-made carbon film indirectly coated on mica. Carbon films can be prepared by evaporating carbon on freshly cleaved mica using a carbon evaporating device.

3. Copper EM grids covered with a perforated carbon film, either home-made or commercial, e.g., Quantifoil (Quantifoil Micro Tools, Jena, Germany) or C-flat (Protochips, Raleigh, NC, USA).

4. Liquid nitrogen storage container for cryogrids.

5. Negative staining solution, e.g., $2 \%(\mathrm{w} / \mathrm{v})$ uranyl formate in water (see Note 3 ).

6. Glycerol-free buffer: $20 \mathrm{mM}$ HEPES-KOH pH 7.5, $30 \mathrm{mM}$ $\mathrm{KCl}, 10 \mathrm{mM} \mathrm{Mg}(\mathrm{OAc})_{2}, 0.5 \mathrm{mM}$ DTT.

1. Freeze-plunging device (home-made or commercial, e.g., Vitrobot, FEI, Eindhoven, The Netherlands or Cryoplunge, Gatan, Pleasanton, CA, USA). See Note 4.

2. Glow-discharging device (home-made or Leica Microsystems, Wetzlar, Germany).

3. Buffer-exchange columns, e.g., Zeba spin columns (Pierce, Rockford, IL, USA).

4. Liquid $\mathrm{N}_{2}$. Gaseous ethane. See Note 4 . 
2.7. Electron

Microscopy

and Single-Particle Image Processing
5. Copper EM grids covered with a perforated carbon film, either home-made or commercial, e.g., Quantifoil (Quantifoil Micro Tools) or C-flat (Protochips).

6. Liquid nitrogen storage container for cryogrids.

1. Electron cryomicroscope equipped with $\mathrm{LaB}_{6}$ cathode or field emission gun (FEG), suitable lens, cryostage, and low-dose capabilities (e.g., FEI or JEOL, Tokyo, Japan).

2. For imaging at room temperature: Room temperature sideentry holder or loading system (e.g., FEI or JEOL).

3. For imaging under cryogenic conditions: Side-entry cryoholder (Gatan) or cryoloading system (FEI).

4. For imaging on a charge-coupled device (CCD) camera: Slow-scan CCD camera optimized for low-dose work (e.g., $4 \mathrm{k} \times 4 \mathrm{k}$ CCD camera from Tietz, Gauting, Germany or Gatan, or FEI).

5. For imaging on photographic film: Kodak SOl63 photographic film.

6. For imaging on photographic film: High-resolution scanner, e.g., Tango, Heidelberger Druckmaschinen, Heidelberg, Germany or CoolScan, Nikon, Tokyo, Japan.

7. Computer cluster for image processing and graphical workstation for particle selection, visualization of single-particle image processing intermediates, and 3D maps.

8. Image processing software, e.g., IMAGIC (http://www. imagescience.de), SPIDER (http://www.wadsworth.org/ spider_doc/spider/docs/spider.html), EMAN (http://blake. bcm.tmc.edu/eman/emanl), XMIPP (http://xmipp.cnb. csic.es), or FREALIGN (http://emlab.rose2.brandeis.edu/ grigorieff/downloads.html).

9. 3D visualization software, e.g., Amira (www.amiravis.com), Chimera (http://www.cgl.ucsf.edu/chimera/download. html), PyMOL (http://pymol.sourceforge.net).

\section{Methods}

\subsection{TAP Purification of RNA Editing Complexes}

Native RNA editing complexes were isolated by tandem-affinity purification (TAP) (12). For that we generated a trypanosome cell line that expresses a C-terminally TAP-tagged version of the mitochondrial protein TbMP42, which is an integral component of the Trypanosoma brucei editosome $(13,14)$. The open reading frame was amplified from genomic DNA of T. brucei strain 427 (15). The resulting PCR fragment was cloned into pLEW100/TAP, 
a derivative of pLEW100 (11). pLEW100/TAP contains the TAP-tag cassette of pBS1479 (12). The expression plasmid pLEW-TbMP42/TAP was transfected into T. brucei strain 29-13 to allow conditional expression of TbMP42/TAP by adding tetracyclin (tet) to the culture medium (11). Clonal cell lines of 29-13-MP42/TAP were established by limited dilution. Expression of the $64.3 \mathrm{kDa}$ (577 amino acids) TbMP42/TAP protein was verified by Western blotting.

1. Grow 29-13 TbMP42/TAP trypanosomes in SDM-79 medium supplemented with $10 \%(\mathrm{v} / \mathrm{v})$ FCS, $7.5 \mathrm{mg} / 1$ hemin, and $50 \mathrm{U} / \mathrm{ml}$ penicillin/streptomycin at $27^{\circ} \mathrm{C}$.

2. At a cell density of $1-2 \times 10^{7}$ cells $/ \mathrm{ml}$ induce expression of TbMP42/TAP by adding $1 \mu \mathrm{g} / \mathrm{ml}$ tetracycline to the culture medium. Incubate for 72-96 h.

3. Harvest parasites from 201 of cell culture at cell densities between 1 and $2 \times 10^{7}$ cells $/ \mathrm{ml}$.

4. Isolate mitochondrial vesicles at isotonic conditions by $\mathrm{N}_{2}$ cavitation (16).

5. Lyse vesicle preparations in $10 \mathrm{ml}$ editing buffer containing $0.6 \%(\mathrm{v} / \mathrm{v})$ Nonidet-P40 for $30 \mathrm{~min}$ at $4^{\circ} \mathrm{C}$ and spin clear the lysate.

6. Equilibrate $1 \mathrm{ml}$ of $\operatorname{IgG}$ sepharose beads (binding capacity: $1 \mathrm{mg} / \mathrm{ml}$ ) in $10 \mathrm{ml} \mathrm{IPP150.}$

7. Add 2 volumes of IPP150, the equilibrated IgG sepharose beads, and $5 \mathrm{U} \mathrm{RNasin}{ }^{\odot}$ to the cleared lysate and incubate for $\mathrm{l}$ h at $4^{\circ} \mathrm{C}$. Transfer beads to a polypropylene column.

8. Wash IgG sepharose beads with $30 \mathrm{ml}$ of IPP150 and equilibrate beads with $10 \mathrm{ml}$ of TEV cleavage buffer.

9. Add $10 \mathrm{ml}$ of TEV cleavage buffer, $5 \mu \mathrm{g} / \mathrm{ml}$ TEV protease, and $5 \mathrm{U} / \mathrm{ml} \mathrm{RNasin}{ }^{\odot}$ to the IgG sepharose beads and incubate for $2 \mathrm{~h}$ at $16^{\circ} \mathrm{C}$.

10. Equilibrate $0.5 \mathrm{ml}$ of calmodulin affinity resin (binding capacity: $1 \mathrm{mg} / \mathrm{ml}$ ) in $10 \mathrm{ml}$ calmodulin binding buffer.

11. Collect the TEV eluate and add 2 volumes of calmodulin binding buffer, calmodulin affinity resin, and $5 \mathrm{U} / \mathrm{ml} \mathrm{RNasin(C)}$ Incubate for $1 \mathrm{~h}$ at $4^{\circ} \mathrm{C}$.

12. Wash calmodulin affinity resin with $30 \mathrm{ml}$ of calmodulin binding buffer.

13. Elute with calmodulin elution buffer in three $1 \mathrm{ml}$ fractions.

14. Analyze an aliquot of each elution fraction in a $10 \%(\mathrm{w} / \mathrm{v})$ SDScontaining polyacrylamide gel. Visualize by silver staining.

The TAP eluate was tested for its RNA editing activity using the precleaved RNA editing assays $(17,18)$. To analyze the TAP 
eluate on the protein level, protein bands were excised from SDS-containing polyacrylamide gels and "in gel"-digested with trypsin. Peptides were identified by mass spectrometry (MS). The MS spectra were analyzed using MS-Fit (19).

Apparent sedimentation coefficients ( $\mathrm{S}$-values) of the purified complexes were determined by isokinetic density centrifugation in linear glycerol gradients. To increase the detection limit, RNA editing complexes were radioactively labeled by protein iodination (20). In the presence of Chloramine-T, sodium iodide $\left(\mathrm{Na}^{125} \mathrm{I}\right)$ is oxidized to molecular $\mathrm{I}_{2}$ or $\mathrm{ICl}$. Both molecules react specifically with tyrosines and to a lesser degree with histidines to form stable, covalent protein- ${ }^{125} \mathrm{I}$ bonds.

1. Add $3.7 \mathrm{MBq} \mathrm{Na}{ }^{125} \mathrm{I}(74 \mathrm{TBq} / \mathrm{mmol})$ to $0.5 \mathrm{mg}$ TAP eluate and transfer the sample to a fume hood (see Note 1). Start the reaction by adding $10 \mu \mathrm{l}$ Chloramine- $\mathrm{T}(2.5 \mathrm{mg} / \mathrm{ml})$ freshly dissolved in $50 \mathrm{mM}$ sodium phosphate, $\mathrm{pH}$ 7.5. Incubate for $2 \mathrm{~min}$ at room temperature.

2. Quench the reaction by adding $20 \mu \mathrm{l}$ of $3 \mathrm{mg} / \mathrm{ml} \mathrm{Na}_{2} \mathrm{~S}_{2} \mathrm{O}_{5}$ freshly dissolved in $50 \mathrm{mM}$ sodium phosphate, $\mathrm{pH}$ 7.5.

3. Separate radioactively labeled complexes in a $2.2 \mathrm{ml} 0-40 \%$ $(\mathrm{v} / \mathrm{v})$ glycerol gradient for $2 \mathrm{~h}$ at $100,000 \times \mathrm{g}$ at $4^{\circ} \mathrm{C}($ Beckman TLS55 rotor).

4. Collect eleven $0.2 \mathrm{ml}$ fractions from the top of the gradient.

5. Quantify gradient fractions by TCA precipitation followed by scintillation counting.

6. Determine the glycerol concentration of the gradient fractions by measuring the refractive index. To correlate a specific refractive index with an apparent $S$-value, marker molecules (5S rRNA, thyroglobin (19S), 23S rRNA, 30S and 50S ribosomal subunits) should be run on a separate gradient.

3.2. Surface Plasmon Resonance
Quantitative data for the interaction of mRNA, gRNA, and mRNA/gRNA hybrid molecules with 20 S editosomes can be derived from SPR experiments.

For a covalent attachment of the RNA to the SPRmicrocuvette, $3^{\prime}$-oxidized RNA was bound to the surface of an amino silane microcuvette. In the presence of $\mathrm{NaIO}_{4}, \mathrm{RNA}$ is oxidized at the $2^{\prime}$ and $3^{\prime}$ position of the $3^{\prime}$ terminal ribose ring by conversion of the hydroxyl groups to aldehyde groups. The aldehyde groups form Schiff's bases with the primary amines of the aminosilane surface, which can be reduced to secondary amines.

1. For the $3^{\prime}$ oxidation, incubate $10 \mu \mathrm{g}$ of RNA with $20 \mathrm{mM}$ $\mathrm{NaIO}_{4}$ in oxidation buffer for $3 \mathrm{~h}$ at $4^{\circ} \mathrm{C}$ in the dark.

2. Purify oxidized RNA by gel filtration. 
3. Insert an aminosilan-coated microcuvette into the IAsys instrument and add the oxidized RNA in $200 \mu \mathrm{l}$ of coupling buffer. Incubate for $3 \mathrm{~h}$ at $27^{\circ} \mathrm{C}$ without stirring. Monitor the coupling reaction in real time.

4. Wash the cuvette $3 \times$ for $10 \mathrm{~min}$ with editing buffer.

The interaction of editing complexes with the RNA surface can be monitored in real time by SPR. All steps should be performed at $27^{\circ} \mathrm{C}$ with a stirrer frequency of $80 \mathrm{~Hz}$. All buffers must be prewarmed to $27^{\circ} \mathrm{C}$ to avoid fluctuations in the resonant angle caused by a temperature shift.

1. Wash the cuvette with editing buffer until a stable baseline is reached.

2. Add a known editosome concentration (2-40 nM). An interaction of the RNA editing complexes with the RNA surface results in an increase of the resonant angle until saturation is reached. Typically, binding is completed within 1-2 min.

3 . Add $3 \times 200 \mu$ l editing buffer. Dissociation of editosomes from the RNA surface is achieved by dilution with excess of binding buffer. Dissociation results in a decrease of the resonant angle.

4. Regenerate the surface by adding $3 \times 200 \mu \mathrm{l} 6 \mathrm{M}$ guanidinium$\mathrm{HCl}$ in editing buffer. This step is required to remove remaining editosomes from the RNA surface.

5. Wash the cuvette $3 \times$ for 10 min with editing buffer and set the baseline. The cuvette is now ready for the next binding event.

6. Determine $k_{\text {diss }}$ and $k_{\text {ass }}$ values by plotting observed on rates $\left(k_{\text {on }(\mathrm{obs})}\right)$ as a function of the complex concentration $\left(k_{\text {on }(\mathrm{obs})}=k_{\text {ass }} \times[\right.$ complex $\left.]+k_{\text {diss }}\right)$. Equilibrium dissociation constants $\left(K_{\mathrm{d}}\right)$ are calculated as $K_{\mathrm{d}}=k_{\text {diss }} / k_{\text {ass }}$.

3.3. GraFix Fractionation
The RNA editing complexes isolated from the mitochondria of trypanosomes using the TAP-tag approach are a mixture of different assemblies (6). Thus, an additional purification step is favorable. To this end, we use gradient ultracentrifugation resulting in a separation of complexes dependent on their sedimentation characteristics. In addition, the complexes are further stabilized by a mild chemical fixation gradient during ultracentrifugation using the GraFix protocol (9). For visualization of the RNA editing complexes by EM, this approach was necessary (see Note 5).

1. Prepare glycerol or sucrose density gradients in a suitable buffer freshly (see Note 6). For RNA editing complexes, use low and high percentage buffers (see Note 2). Use a gradient forming device and keep the gradients at $4^{\circ} \mathrm{C}$ for about $\mathrm{l} \mathrm{h}$. 
2. As an optional step for $13.2 \mathrm{ml}$ gradient tubes, $0.5 \mathrm{ml}$ of cushion buffer can be added prior to sample loading (see Note 7).

3. Carefully load the sample (up to $1.5 \mathrm{ml}$, depending on the sample concentration) on top of the gradient/cushion. Load at least $10-20 \mu \mathrm{g}$ of protein on a $13.2 \mathrm{ml}$ gradient. Overloading of the gradient should be avoided to prevent inter-particle crosslinking.

4. Spin, e.g., for $14 \mathrm{~h}$ at $247,000 \times g$ and $4^{\circ} \mathrm{C}$ using a Sorvall $\mathrm{TH}-641$ rotor.

5. Carefully fractionate the gradient from the bottom at $4^{\circ} \mathrm{C}$ using a needle introduced from the top of the gradient or alternatively use a piercing device (see Note 8). Fractions of 10 drops (corresponding to a volume of about $300 \mu \mathrm{l}$ ) are well suited for a $13.2 \mathrm{ml}$ gradient.

3.4. Negative Staining and Cryo-negative Staining
Negative staining is a standard method to prepare macromolecular complexes for the visualization in an electron microscope; images are taken at room temperature. The approach uses various heavy metal stains such as uranyl salts. Due to the enhanced contrast also particles $<100 \mathrm{kDa}$ can be visualized depending on the shape. Cryo-negative staining combines negative staining with cryopreservation of the sample and a resolution in the subnanometer level can be obtained (21). Several different negative staining and cryo-negative staining protocols have been reported (22-24). We describe here the sandwich carbon procedure for imaging at room temperature and under cryogenic conditions. Both approaches were recently used to visualize endogenous RNA editing complexes isolated from trypanosomes (6).

1. Fill the sample (typically about $25 \mu \mathrm{l}$ ), the staining solution (about 100-200 $\mu \mathrm{l}$ ), and a washing buffer (about 100-200 $\mu \mathrm{l}$, optional step) into the wells of a black plastic or Teflon block. Keep the block throughout the sample preparation at $4^{\circ} \mathrm{C}$ (see Note 9). For uranyl salts, see also Note 3.

2. Carefully place a small piece (approximately $3 \times 3 \mathrm{~mm}$ ) of a continuous carbon film (that has previously been evaporated on mica in an indirect setup of the evaporating device) in the sample solution such that the carbon film detaches from the mica (Fig. la).

3. The incubation time depends on the specific sample and may vary between $15 \mathrm{~s}$ and $48 \mathrm{~h}$. For editosomes, an adsorption time of $0.5-6$ h was typically used.

4. For some samples, a washing step might be useful to increase the image contrast (Fig. Ib). This optional step can be done by transferring and incubating the carbon film for several seconds to few minutes in 100-200 $\mu$ l of a washing buffer in 


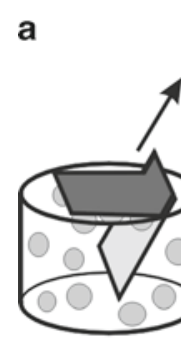

sample b

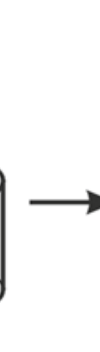

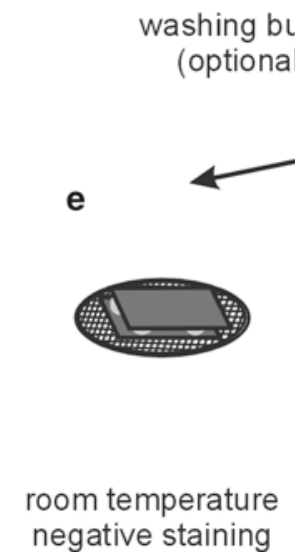

c

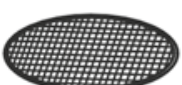

d

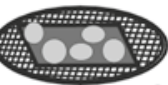

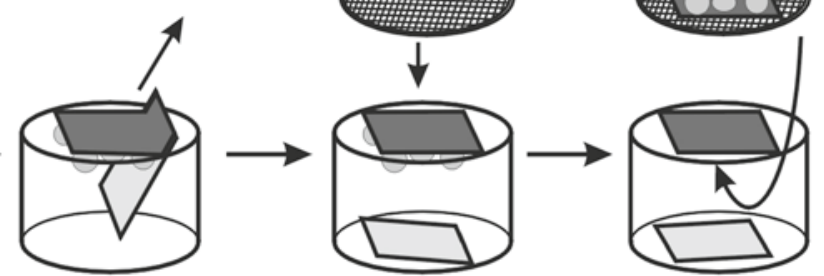

staining solution staining solution

negative staining

Fig. 1. Cryo-negative staining and negative staining. (a) Initially, the sample (particles are depicted as gray spheres) and the staining solution (two wells) are filled into wells of a black plastic or Teflon block. Optionally, wells can be filled with a washing buffer, if necessary. Most of the steps are identical for both room temperature negative staining and cryonegative staining (a-d); the procedures differ only in the final step (e or $\mathbf{f}$ ). A small piece of carbon film (dark gray) that was indirectly evaporated on a piece of mica (light gray) is placed into the sample solution. Thereby, the carbon film detaches from the mica except for a small area where the tweezers are touching it, and the macromolecules can adsorb for a defined period of time. (b) Optionally, the carbon film to which the particles have adsorbed can be transferred to a well filled with a washing buffer to improve the staining quality. This step can also be repeated, if necessary. (c) Subsequent to particle adsorption or the optional washing step, the carbon film is transferred to a well filled with a staining solution. Thereby, the mica completely detaches from the carbon film and falls down to the bottom of the well, while the carbon film with the particles facing towards the staining solution is floating on the surface of the staining solution. A copper EM grid (top) covered with a perforated carbon film is placed on top of the floating carbon film and the EM grid is carefully removed from the staining solution. Excess liquid is blotted from the side without destroying the carbon film. (d) Another piece of carbon film is placed into a second well filled with staining solution so that the carbon film is completely floating on the staining solution. The EM grid with the particles facing up is submerged underneath the second carbon film and lifted out of the staining solution to form the sandwich. Again, excess liquid is blotted. (e) For room temperature negative staining, the grid is dried and can then be stored at a dry place until imaging. (f) For cryo-negative staining, the EM grid is frozen in liquid nitrogen. Cryo grids must be stored and imaged under cryogenic conditions.

exactly the same way as carried out for the particle adsorption step (see Subheading 3.4, step 2).

5. Lift the carbon/mica out of the particle or optional washing solution, blot it carefully without touching the carbon film and transfer it to the staining solution (Fig. lc). The carbon must completely detach from the mica. The particles face down towards the staining solution.

6. After about $2 \mathrm{~min}$, the carbon film is lifted out of the staining solution by putting an EM grid covered with a perforated 
carbon film on top of the floating carbon (Fig. 1c). Submerge the EM grid underneath the staining solution and carefully remove the EM grid out of the staining solution. Blot excess liquid from the side.

7. To form the carbon sandwich, float another piece of carbon film onto the surface of a second well filled with the staining solution so that the mica completely detaches from the carbon film (Fig. 1d). Submerge the EM grid with the particles facing up underneath the floating carbon film and lift the EM grid out of the solution to embed the particles in a layer of staining solution between the two carbon films. Again, blot the EM grid carefully from the side.

8. Let the EM grid air-dry for imaging at room temperature (Fig. le) or freeze the grid in liquid $\mathrm{N}_{2}$ for cryo-negative staining (Fig. lf). Once frozen, the grid must be kept under cryogenic conditions.

3.5. Unstained Cryopreparation
Unstained cryopreparations are obtained by freeze-plunging of the sample into liquid ethane (25). This results in the vitrification of the sample, i.e., the amorphous structure of the buffer is preserved and no crystalline ice is formed. Unstained cryoimaging can be performed for all samples of sufficient concentration and molecular mass (typically $>250 \mathrm{kDa}$ ). In addition, the buffer must be compatible with vitrification. In particular, glycerol, sucrose, and high salt concentrations may interfere with the vitrification. In all such cases (e.g., GraFix fractions), the sample needs to be buffer exchanged to a suitable buffer.

1. Optional step in case substances with an adverse effect on the vitrification are present in the particle buffer: Buffer exchange the GraFix sample for a glycerol-free buffer using a Zeba spin column (see Note 10).

2. Fill 25-30 $\mu \mathrm{l}$ of sample into a well of the black plastic or Teflon block and proceed as described in Subheading 3.4, step 2 . Keep the sample at $4^{\circ} \mathrm{C}$.

3. The adsorption time needs to be adjusted according to the sample and may vary between $15 \mathrm{~s}$ and $48 \mathrm{~h}$.

4. Place a copper EM grid covered with a perforated carbon film on top of the floating carbon film, submerge it underneath the surface, and carefully remove it out of the solution. Prior to usage, the EM grid can be glow discharged.

5. Mount the tweezers holding the EM grid in the freezeplunger filled with liquid ethane (see also Note 4 ). Blot the grid carefully and plunge it into the liquid ethane (see Note 11). The EM grid is transferred and stored in liquid $\mathrm{N}_{2}$ until imaging. 
3.6. Electron

Microscopy and Single-Particle Image Processing
The transmission electron microscope is used to image biological samples in the form of two-dimensional (2D) projection views. Due to the radiation sensitivity of biological material, imaging has to be conducted at low-dose conditions, and a further protection of the sample can be achieved by imaging at cryogenic temperatures (i.e., liquid $\mathrm{N}_{2}$ - or He-cooling) as compared to room temperature (26). To obtain the 3D information out of the data set of $2 \mathrm{D}$ projections, the particle views have to be subjected to single-particle image processing $(7,8)$. An overview of the steps is given in Fig. 2 and the single-particle image processing of the $\sim 20$ S and $~ 35-40$ S RNA editing complexes isolated from T. brucei is summarized in Figs. 3 and 4, respectively.

1. Negatively stained grids can be transferred using standard room temperature holders into the microscope. For cryoimaging, the grid has to be mounted in a specialized side-entry cryoholder or a cryoloading system. During the mounting and transfer of the cryogrids, the sample has to be kept at cryogenic conditions (see Note 12).

2. For de novo structure determinations, a slow-scan CCD camera offers significant advantages compared to conventional photographic film; the latter usually is advantageous for highresolution work (27). See Note 13. The magnification of the electron microscope has to be adjusted depending on, e.g., the size of the object, number of particles, pixel size of the detector, and desired resolution of the images. For CCD camera images, magnifications of about 50,000-275,000fold and for photographic films, magnifications of 27,000 60,000-fold are typically used. Images can be taken untilted or at a tilt angle supported by the cryostage/holder combination. In particular, for the random conical tilt (RCT) technique (28), images are first taken at the selected tilt angle (e.g., $45^{\circ}$ ) and subsequently in an untilted mode at the same position to reduce the beam damage of the tilted images (as the tilted images are used for 3D calculation).

3. For photographic film only: Photographic film has to be digitized with a scanner of appropriate quality (e.g., Tango or Coolscan). Low quality images (e.g., poor contrast, drift, charging) can be discarded prior to this step.

4. Select individual particles from the raw EM images by manual or (semi-) automated procedures (see Note 14). Algorithms for manual and/or (semi-) automated particle selection are implemented in all major single-particle image processing software packages and individual programs are also available (e.g., (29-32)). Automated procedures can be combined with a postselection step in which all positions that were found by the software, but do not show a particle of desired quality are removed. 


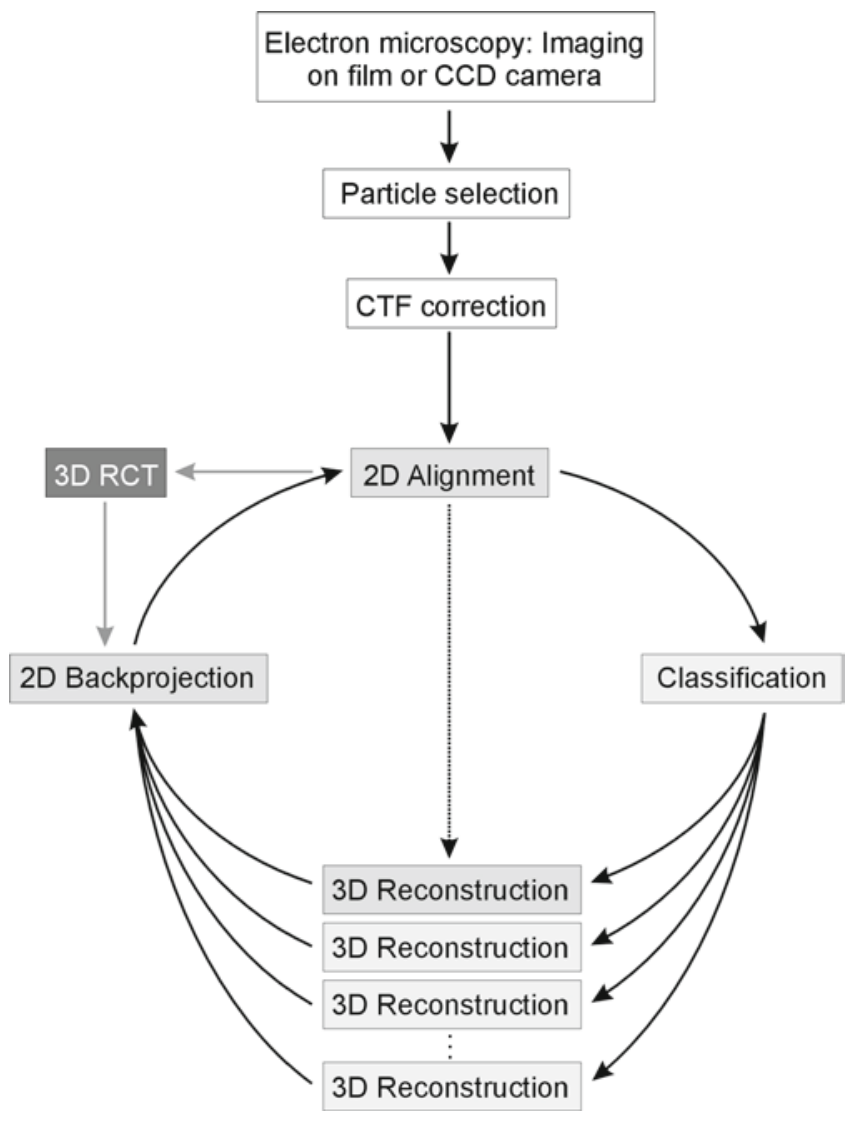

Fig. 2. Schematic representation of the individual steps performed during single-particle image processing. The initial steps (white boxes) comprise the imaging of the macromolecule, particle selection from the raw EM images, and correction for the CTF. The single-particle images are subsequently subjected to iterative rounds of single-particle image processing (labeled "2D Alignment," "Classification," "3D Reconstruction," and "2D Backprojection"). Initial 3D maps of a macromolecular assembly can be calculated using the RCT (labeled "3D RCT") or angular reconstitution approach. During the refinement phase, the alignment process itself can be used for grouping of similar views in alignment averages and these can be used for 3D calculation (dashed arrow). Competitive multireference alignment can be performed for samples of dynamic macromolecular assemblies characterized by the presence of multiple 3D structural states (labeled "3D Reconstruction" in the bottom part of the circle). Thereby, the individual 3D maps are backprojected into the 2D space (labeled "2D Backprojection"; in case of competitive multireference alignment, these 2D backprojections derive from different 3D maps) and each single-particle image is competitively aligned to these reference projections.

5. Correct for the contrast-transfer-function (CTF) imposed on the raw EM images (see Note 15). Programs for this are included in all major software packages as well as in individual programs (e.g., (29-31, 33)). This step also offers the possibility to identify low quality images.

6. The particle images can now be used for iterative rounds of $2 \mathrm{D}$ and $3 \mathrm{D}$ image processing (Fig. 2). This is typically done 
a

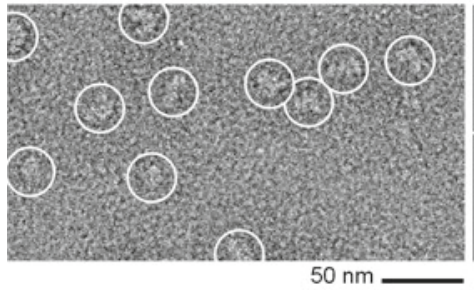

b
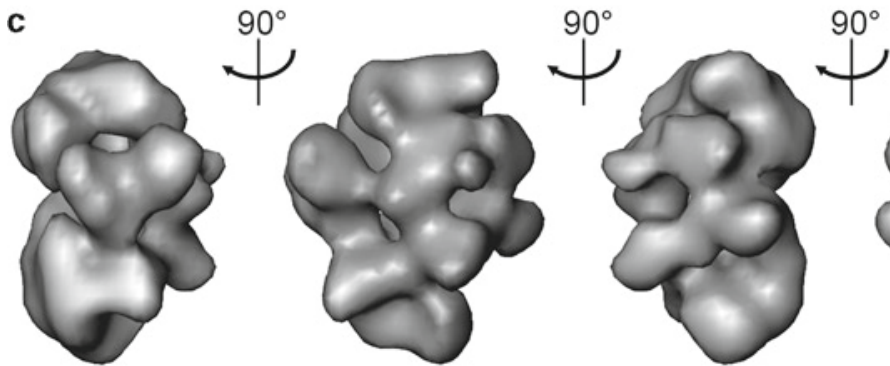

Fig. 3. Single-particle electron microscopy of the 20 S editosome of Trypanosoma brucei (6). (a) Raw electron microscopical image of negatively stained $\sim 20$ S RNA editing complexes. Individual particles are encircled. (b) Class averages of the $\sim 20 \mathrm{~S}$ editosome as obtained upon 2D single-particle image processing. Some of the class averages represent different structural subtypes of the $\sim 20 \mathrm{~S}$ complexes and thus belong to different 3D maps. (c) 3D map of the $\sim 20 \mathrm{~S}$ editosome using cryo-negatively stained images. Subsequent to separation of the data set into structural subtypes by competitive multireference alignment, the 3D structure was determined from a subset of images belonging to one structural subtype of the $\sim 20 \mathrm{~S}$ editosome. 3D views were slightly rotated with respect to the published orientations (6).

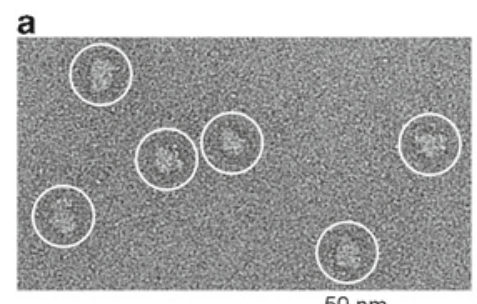

$50 \mathrm{~nm}$ b

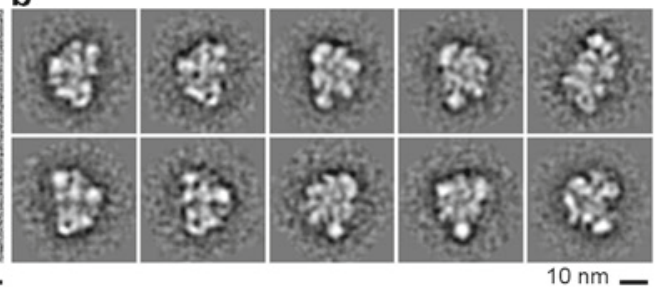

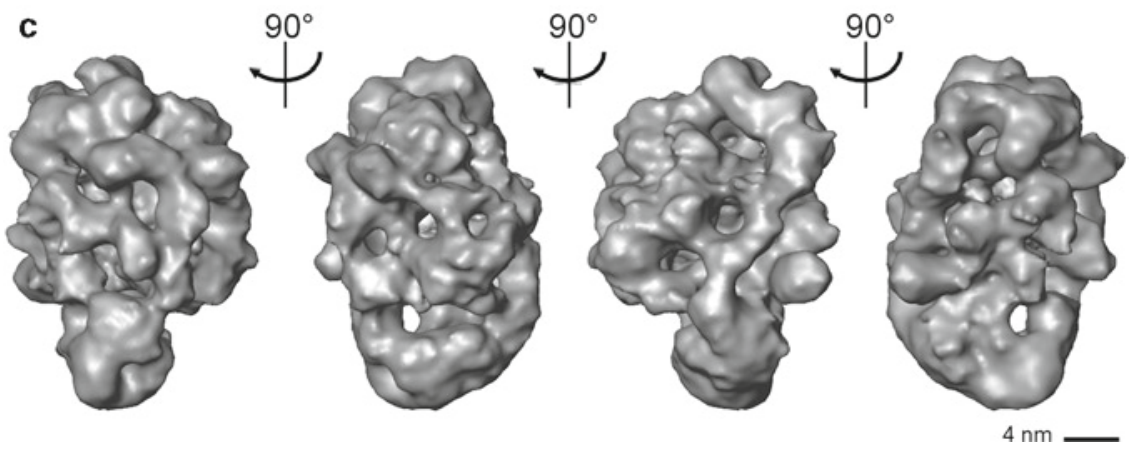

Fig. 4. Single-particle electron microscopy of the 35-40S editosome of Trypanosomal brucei (6). (a) Raw electron microscopical image of negatively stained $\sim 35-40 S$ RNA editing complexes. Individual particles are encircled. (b) Class averages of the $\sim 35-40 S$ editosome as obtained upon 2D single-particle image processing. Some of the class averages represent different structural subtypes of the $\sim 35-40$ S complexes and thus belong to different 3D maps. (c) 3D map of the $\sim 35-40 S$ editosome using cryo-negatively stained images. Subsequent to separation of the data set into structural subtypes by competitive multireference alignment, the 3D structure was determined from a subset of images belonging to one structural subtype of the $\sim 35-40$ S editosome. 3D views were slightly rotated with respect to the published orientations (6). 
in one of the major software packages such as, e.g., IMAGIC (29), SPIDER(30), EMAN (31), XMIPP (34), or FREALIGN (35) (see Note 16). One round of 2D image processing typically comprises of an alignment step and a classification step. During the alignment, the particle images are aligned towards references to bring similar views into register (see Note 17). These aligned images can subsequently be used to group similar views into classes in order to increase the signal-to-noiseratio. Alignment and classification are iteratively repeated until the result is stable.

7. The class or alignment averages are then used to calculate the $3 \mathrm{D}$ map of the macromolecular assembly. For this, all programs listed above can be used. Several different techniques exist including, e.g., RCT (28), angular reconstitution (36), and projection matching (37) (see Note 18).

8. For a refinement of the initial model (e.g., a low-resolution 3D map determined using the RCT or angular reconstitution approach), the Subheading 3.6, steps 6 and 7 are repeated until the result is stable and no improvement of the structure is observed. 2D backprojections of the 3D map are used as an alignment reference during the refinement of the 3D map. For dynamic macromolecular assemblies, several 3D structural maps representing different compositional and/or conformational states can be determined using the RCT technique. These different 3D maps can be used to generate sets of $2 \mathrm{D}$ backprojections for a competitive multireference alignment. Each single-particle image is thereby competitively aligned to these reference projections, and the data set is separated into subsets representing individual structural subtypes. These individual structural subtypes are refined separately. Typically, during the refinement steps, a large number of references is used to cover all possible angular directions. This makes more advanced schemes such as the corrims (38) necessary to speedup the calculation process.

9. Finally, the $3 \mathrm{D}$ map is visualized as sections and/or surface views in a $3 \mathrm{D}$ viewer and can be annotated by, e.g., fitting of substructures, labeling, recognition of structural elements, and other techniques.

\section{Notes}

1. ${ }^{125} \mathrm{I}$ emits gamma rays with a maximal energy of $0.035 \mathrm{MeV}$ and requires lead shielding. Unbound iodine is volatile and must be handled under a fume hood. 
2. We recommend using fresh EM-grade solutions of $25 \%(\mathrm{v} / \mathrm{v})$ glutaraldehyde since the activity of the crosslinker may decrease over time. Glutaraldehyde and other crosslinkers are highly toxic and appropriate safety precautions according to the manufacturer should be followed.

3. Prepare the uranyl formate solution always freshly and keep the solution cool and in the dark. Uranyl salts are radioactive and toxic and thus appropriate safety precautions should be followed.

4. Liquid $\mathrm{N}_{2}$ and liquid ethane can cause severe burns. Note the asphyxiation hazard. Ethane is extremely flammable and forms explosive mixtures with air. Appropriate protection is required.

5. Depending on the particle, GraFix can have a number of advantages: (1) significant reduction of particle disintegration and aggregation, (2) improved adsorption towards the carbon support film, (3) increased image contrast, and (4) increase in angular diversity.

6. As the gradient contains a low amount of glutaraldehyde, only buffer reagents compatible with the fixation reagent can be used. Glutaraldehyde, for example, reacts with primary amines $(39,40)$, and thus all gradient buffers must be free of primary amines.

7. Some purification protocols result in the presence of primary amine compounds in the sample in addition to the protein or RNA/protein complex of interest. These include, e.g., elution peptides contaminating polypeptides at high concentration and Tris-OH. As a direct contact of such a sample with the crosslinker has to be avoided, a cushion free of primary amines and free of glutaraldehyde is used before loading the sample.

8. We highly recommend fractionating the gradient from the bottom since low molecular mass substances such as peptides and detergents are enriched in the top fractions of the gradient. These low molecular mass substances may interfere with the image contrast of the EM specimen and may cause staining artifacts. Using a needle/tube system connected to a peristaltic pump, mixing of the gradient, e.g., during the insertion of the needle has to be avoided.

9. During long-term incubation, the block has to be kept at $4^{\circ} \mathrm{C}$ and condensation of water vapor on the sample must be avoided. This can be accomplished by placing the block into a dry, precooled Petri dish, mounting the lid and storing the block/Petri dish in a cold room. Even during short-term incubation, the block must be kept at $4^{\circ} \mathrm{C}$ and condensation of water vapor must be avoided. However, it is usually sufficient to place the block in an ice-cooled Petri dish. 
10. As the recovery of the sample upon buffer exchange may vary with the properties of the selected particle (for large multimegadalton assemblies in the range of about 20-95\%), we recommend to prepare a negative staining room temperature EM grid to adapt the incubation time for the unstained cryopreparation accordingly.

11. The blotting properties (i.e., time, pressure, one-sided vs. two-sided, frequency, etc.) as well as the environmental properties (temperature and humidity) have to be adapted to the specific sample under investigation. Assessment of the vitrification quality must be done by using the electron cryomicroscope under cryoconditions.

12. Mounting and transfer of room temperature EM grids is straightforward. Significant warming and ice contamination of cryo-EM grids during the mounting and transfer under cryogenic conditions must be avoided.

13. Slow-scan CCD cameras offer an increased phase transmission and spectral signal-to-noise ratio (27), which are important factors in the setup of a novel structure. Images can be recorded in tile mode - i.e., with slight overlap of the images and stitched to larger images to compensate for the smaller imaging area of the CCD camera. In contrast, photographic film shows superior quality in the very high resolution range.

14. Manual selection of particles is more time-intensive compared to (semi-) automated selection procedures, but offers the opportunity to get an overview about the typical views and the quality of the imaged particles (e.g., homogeneity, aggregation, disintegration). In particular for particles with so-far unknown structure, manual selection is advantageous. For the selection of larger data sets of particles with known structure, automated particle selection can be performed using "Boxer" (EMAN software package (31)) or "Signature" (32). Manual postselection is often recommended to remove low quality images (e.g., aggregates, broken particles, ice contamination). In any of the methods, the particles should be picked centrally, i.e., a displacement of the particle in direction of the edges should be avoided. Particles are extracted from the raw images in a pixel frame larger than the actual maximum dimensions of the visualized particles. In general, a pixel frame in which the particles amount to about two thirds of the frame is recommended. Depending on the particle dimensions and the pixel size, typical values of the pixel frame are $64 \times 64$ to $512 \times 512$ pixels.

15. Computationally, raw EM images should be corrected for the defocus and twofold astigmatism, and can also be corrected for the experimental B factor, if desired. Low quality images due to drift or charging can be easily identified in the $2 \mathrm{D}$ power spectra as truncations of the Thon rings. 
16. File format type and definition of the coordinate system including 3D angles may vary between the individual programs and thus need to be checked carefully when switching from one software package to the other.

17. There are several ways to generate reference images for the alignment. In case of a particle with an unknown structure, selected single-particle views or the sum of all images (i.e., a featureless "blob") can be used as a first reference. Also, views from a related particle (e.g., with minor differences in composition) can be used in the first alignment. For subsequent rounds, class or alignment averages can be used (see below). Once a 3D model of the analyzed or a related (e.g., with minor differences in composition) particle is available, this can be used to generate $2 \mathrm{D}$ projections by backprojecting the $3 \mathrm{D}$ map into 2D projections. For averaging, either a statistical classification approach such as multivariate statistical classification or - in particular in the advanced steps of the refinement - the alignment itself is used. The former technique generates so-called class averages, the latter alignment averages.

18. All of the different $3 \mathrm{D}$ reconstruction techniques are implemented in one or more of the software packages listed in Subheading 2.7, item 8 . The selection of the approach primarily depends on the stage of the project. RCT (28) and angular reconstitution (36) can be used to determine the structure of a particle whose structure was so-far unknown, i.e., in a de novo approach. For refinement of an available model, angular reconstitution and projection matching (37) can be used. RCT structures are typically limited to the low resolution range and suffer from a missing cone, but in contrast to the other techniques can determine the handedness of the complex. Refinement of an RCT structure is possible by both angular reconstitution and projection matching. Angular reconstitution and projection matching can reconstruct $3 \mathrm{D}$ maps to the subnanometer level.

\section{Acknowledgments}

MMG and BS are supported by a grant from the Danish Center for Scientific Computing (DCSC). HS is supported by a grant of the Bundesministerium für Bildung und Forschung (BMBF) and a European "3D Repertoire" grant. HUG is supported as an International Scholar of the Howard Hughes Medical Institute (HHMI) and by the German Research Foundation (DFG). 


\section{References}

1. Alberts, B. (1998) The cell as a collection of protein machines: preparing the next generation of molecular biologists Cell 92, 291-4.

2. Madison-Antenucci, S., Grams, J., and Hajduk, S. L. (2002) Editing machines: the complexities of trypanosome RNA editing Cell 108, 435-8.

3. Stuart, K. D., Schnaufer, A., Ernst, N. L., and Panigrahi, A. K. (2005) Complex management: RNA editing in trypanosomes Trends Biochem Sci 30, 97-105.

4. Stark, H., and Lührmann, R. (2006) Cryoelectron microscopy of spliceosomal components Annu Rev Biophys Biomol Struct 35, 435-57.

5. Leschziner, A. E., and Nogales, E. (2007) Visualizing flexibility at molecular resolution: analysis of heterogeneity in single-particle electron microscopy reconstructions Annu Rev Biophys Biomol Struct 36, 43-62.

6. Golas, M. M., Böhm, C., Sander, B., Effenberger, K., Brecht, M., Stark, H., and Göringer, H. U. (2009) Snapshots of the RNA editing machine in trypanosomes captured at different assembly stages in vivo EMBO J 28, 766-78.

7. van Heel, M., Gowen, B., Matadeen, R., Orlova, E. V., Finn, R., Pape, T., Cohen, D., Stark, H., Schmidt, R., Schatz, M., and Patwardhan, A. (2000) Single-particle electron cryo-microscopy: towards atomic resolution QRev Biophys 33, 307-69.

8. Frank, J. (2002) Single-particle imaging of macromolecules by cryo-electron microscopy Annu Rev Biophys Biomol Struct 31, 303-19.

9. Kastner, B., Fischer, N., Golas, M. M., Sander, B., Dube, P., Boehringer, D., Hartmuth, K., Deckert, J., Hauer, F., Wolf, E., Uchtenhagen, H., Urlaub, H., Herzog, F., Peters, J. M., Poerschke, D., Lührmann, R., and Stark, H. (2008) GraFix: sample preparation for singleparticle electron cryomicroscopy Nat Methods $5,53-5$.

10. Brun, R., and Schönenberger, M. (1979) Cultivation and in vitro cloning of procyclic culture forms of Trypanosoma brucei in a semidefined medium Acta Trop 36, 289-92.

11. Wirtz, E., Leal, S., Ochatt, C., and Cross, G. A. (1999) A tightly regulated inducible expression system for conditional gene knock-outs and dominant-negative genetics in Trypanosoma brucei Mol Biochem Parasitol 99, 89-101.

12. Rigaut, G., Shevchenko, A., Rutz, B., Wilm, M., Mann, M., and Seraphin, B. (1999) A generic protein purification method for protein complex characterization and proteome exploration Nat Biotechnol 17, 1030-2.

13. Brecht, M., Niemann, M., Schlüter, E., Müller, U. F., Stuart, K., and Göringer, H. U. (2005) TbMP42, a protein component of the RNA editing complex in African trypanosomes, has endo-exoribonuclease activity $\mathrm{Mol}$ Cell 17, 621-30.

14. Panigrahi, A. K., Schnaufer, A., Carmean, N., Igo, R. P., Jr., Gygi, S. P., Ernst, N. L., Palazzo, S. S., Weston, D. S., Aebersold, R., Salavati, R., and Stuart, K. D. (2001) Four related proteins of the Trypanosoma brucei RNA editing complex Mol Cell Biol 21, 6833-40.

15. Cross, G. A. (1975) Identification, purification and properties of clone-specific glycoprotein antigens constituting the surface coat of Trypanosoma brucei Parasitology 71, 393-417.

16. Hauser, R., Pypaert, M., Hausler, T., Horn, E. K., and Schneider, A. (1996) In vitro import of proteins into mitochondria of Trypanosoma brucei and Leishmania tarentolae J Cell Sci 109 (Pt 2), 517-23.

17. Igo, R. P., Jr., Palazzo, S. S., Burgess, M. L., Panigrahi, A. K., and Stuart, K. (2000) Uridylate addition and RNA ligation contribute to the specificity of kinetoplastid insertion RNA editing Mol Cell Biol 20, 8447-57.

18. Igo, R. P., Jr., Weston, D. S., Ernst, N. L., Panigrahi, A. K., Salavati, R., and Stuart, K. (2002) Role of uridylate-specific exoribonuclease activity in Trypanosoma brucei RNA editing Eukaryot Cell 1, 112-8.

19. Clauser, K. R., Baker, P., and Burlingame, A. L. (1999) Role of accurate mass measurement $(+/-10 \mathrm{ppm})$ in protein identification strategies employing MS or MS/MS and database searching Anal Chem 71, 2871-82.

20. Hunter, W. M., and Greenwood, F. C. (1962) Preparation of iodine-131 labelled human growth hormone of high specific activity Nature 194, 495-6.

21. Golas, M. M., Sander, B., Will, C. L., Lührmann, R., and Stark, H. (2003) Molecular architecture of the multiprotein splicing factor SF3b Science 300, 980-4.

22. Harris, J. R. (2007) Negative staining of thinly spread biological samples Methods $\mathrm{Mol}$ Biol 369, 107-42.

23. Adrian, M., Dubochet, J., Fuller, S. D., and Harris, J. R. (1998) Cryo-negative staining Micron 29, 145-60. 
24. Golas, M. M., Sander, B., Will, C. L., Lührmann, R., and Stark, H. (2005) Major conformational change in the complex SF3b upon integration into the spliceosomal U11/ U12 di-snRNP as revealed by electron cryomicroscopy Mol Cell 17, 869-83.

25. Adrian, M., Dubochet, J., Lepault, J., and McDowall, A. W. (1984) Cryo-electron microscopy of viruses Nature 308, 32-6.

26. Chiu, W., Downing, K. H., Dubochet, J., Glaeser, R. M., Heide, H. G., Knapek, E., Kopf, D. A., Lamvik, M. K., Lepault, J., Robertson, J. D., Zeitler, E., and Zemlin, F. (1986) Cryoprotection in electron microscopy J Microsc 141, 385-91.

27. Sander, B., Golas, M. M., and Stark, H. (2005) Advantages of CCD detectors for de novo three-dimensional structure determination in single-particle electron microscopy J Struct Biol 151, 92-105.

28. Radermacher, M. (1988) Three-dimensional reconstruction of single particles from random and nonrandom tilt series $J$ Electron Microsc Tech 9, 359-94.

29. van Heel, M., Harauz, G., Orlova, E. V., Schmidt, R., and Schatz, M. (1996) A new generation of the IMAGIC image processing system J Struct Biol 116, 17-24.

30. Shaikh, T. R., Gao, H., Baxter, W. T., Asturias, F. J., Boisset, N., Leith, A., and Frank, J. (2008) SPIDER image processing for singleparticle reconstruction of biological macromolecules from electron micrographs Nat Protoc 3, 1941-74.

31. Tang, G., Peng, L., Baldwin, P. R., Mann, D. S., Jiang, W., Rees, I., and Ludtke, S. J. (2007) EMAN2: an extensible image processing suite for electron microscopy J Struct Biol 157, 38-46.
32. Chen, J. Z., and Grigorieff, N. (2007) SIGNATURE: a single-particle selection system for molecular electron microscopy J Struct Biol 157, 168-73.

33. Sander, B., Golas, M. M., and Stark, H. (2003) Automatic CTF correction for single particles based upon multivariate statistical analysis of individual power spectra J Struct Biol 142, 392-401.

34. Sorzano, C. O., Marabini, R., VelazquezMuriel, J., Bilbao-Castro, J. R., Scheres, S. H., Carazo, J. M., and Pascual-Montano, A. (2004) XMIPP: a new generation of an opensource image processing package for electron microscopy J Struct Biol 148, 194-204.

35. Grigorieff, N. (2007) FREALIGN: high-resolution refinement of single particle structures J Struct Biol 157, 117-25.

36. van Heel, M. (1987) Angular reconstitution: a posteriori assignment of projection directions for $3 \mathrm{D}$ reconstruction Ultramicroscopy 21, 111-23.

37. Penczek, P. A., Grassucci, R. A., and Frank, J. (1994) The ribosome at improved resolution: new techniques for merging and orientation refinement in 3D cryo-electron microscopy of biological particles Ultramicroscopy 53, 251-70.

38. Sander, B., Golas, M. M., and Stark, H. (2003) Corrim-based alignment for improved speed in single-particle image processing J Struct Biol 143, 219-28.

39. Hopwood, D. (1972) Theoretical and practical aspects of glutaraldehyde fixation Histochem J 4, 267-303.

40. Migneault, I., Dartiguenave, C., Bertrand, M.J., andWaldron, K.C.(2004) Glutaraldehyde: behavior in aqueous solution, reaction with proteins, and application to enzyme crosslinking Biotechniques 37, 790-6, 8-802. 